University of Wollongong

Research Online

Faculty of Engineering and Information

Faculty of Engineering and Information

Sciences - Papers: Part A

Sciences

2012

Electron emission from amorphous solid water after proton impact: Benchmarking PTra and Geant4 track structure Monte Carlo simulations

M U. Bug

University of Wollongong, mb355@uowmail.edu.au

H Rabus

Physikalisch-Technische Bundesanstalt Germany

Anatoly B. Rosenfeld

University of Wollongong, anatoly@uow.edu.au

Follow this and additional works at: https://ro.uow.edu.au/eispapers

Part of the Engineering Commons, and the Science and Technology Studies Commons

Research Online is the open access institutional repository for the University of Wollongong. For further information contact the UOW Library: research-pubs@uow.edu.au 


\title{
Electron emission from amorphous solid water after proton impact: Benchmarking PTra and Geant4 track structure Monte Carlo simulations
}

\begin{abstract}
Track structure Monte Carlo simulations of ionising radiation in water are often used to estimate radiation damage to DNA. For this purpose, an accurate simulation of the transport of densely ionising low-energy secondary electrons is particularly important, but is impaired by a high uncertainty of the required physical interaction cross section data of liquid water.

A possible tool for the verification of the secondary electron transport in a track structure simulation has been suggested by Toburen et al. (2010), who have measured the angle-dependent energy spectra of electrons, emitted from a thin layer of amorphous solid water (ASW) upon a passage of 6. MeV protons.

In this work, simulations were performed for the setup of their experiment, using the PTB Track structure code (PTra) and Geant4-DNA. To enable electron transport below the ionisation threshold, additional excitation and dissociative attachment anion states were included in PTra and activated in Geant4. Additionally, a surface potential was considered in both simulations, such that the escape probability for an electron is dependent on its energy and impact angle at the ASW/vacuum interface.

For vanishing surface potential, the simulated spectra are in good agreement with the measured spectra for energies above 50. eV. Below, the simulations overestimate the yield of electrons by a factor up to 4 (PTra) or 7 (Geant4-DNA), which is still a better agreement than obtained in previous simulations of this experimental situation. The agreement of the simulations with experimental data was significantly improved by using a step-like increase of the potential energy at the ASW surface. 2012 Elsevier Ltd.
\end{abstract}

\section{Keywords}

after, water, simulations, solid, carlo, amorphous, emission, electron, monte, structure, track, geant4, ptra, benchmarking, impact, proton

Disciplines

Engineering | Science and Technology Studies

\section{Publication Details}

Bug, M. U., Rabus, H. \& Rosenfeld, A. B. (2012). Electron emission from amorphous solid water after proton impact: Benchmarking PTra and Geant4 track structure Monte Carlo simulations. Radiation Physics and Chemistry, 81 (12), 1804-1812. 


\title{
Electron emission from amorphous solid water after proton impact: benchmarking PTra and Geant4 track structure Monte Carlo simulations
}

\author{
M. U. Bug ${ }^{1,2, *}$, H. Rabus ${ }^{1}$, A. B. Rosenfeld ${ }^{2}$ \\ ${ }^{1}$ Physikalisch-Technische Bundesanstalt (PTB), Bundesallee 100, 38116 Braunschweig, Germany \\ ${ }^{2}$ Centre for Medical Radiation Physics, University of Wollongong, Wollongong NSW 2522, Australia \\ * corresponding author
}

\begin{abstract}
Track structure Monte Carlo simulations of ionising radiation in water are often used to estimate radiation damage to DNA. For this purpose, an accurate simulation of the transport of densely ionising low-energy secondary electrons is particularly important, but is impaired by a high uncertainty of the required physical interaction cross section data of liquid water.

A possible tool for the verification of the secondary electron transport in a track structure simulation has been suggested by Toburen et al. (2010), who have measured the angle-dependent energy spectra of electrons, emitted from a thin layer of amorphous solid water (ASW) upon a passage of $6 \mathrm{MeV}$ protons.

In this work, simulations were performed for the setup of their experiment, using the PTB Track structure code (PTra) and Geant4-DNA. To enable electron transport below the ionisation threshold, additional excitation and dissociative attachment anion states were included in PTra and activated in Geant4. Additionally, a surface potential was considered in both simulations, such that the escape probability for an electron is dependent on its energy and impact angle at the ASW/vacuum interface.

For vanishing surface potential, the simulated spectra are in good agreement with the measured spectra for energies above $50 \mathrm{eV}$. Below, the simulations overestimate the yield of electrons by a factor up to 4 (PTra) or 7 (Geant4-DNA), which is still a better agreement than obtained in previous simulations of this experimental situation. The agreement of the simulations with experimental data was significantly improved by using a step-like increase of the potential energy at the ASW surface.
\end{abstract}

Keywords: Track structure Monte Carlo simulation, electron transport, Geant4, PTra, liquid water 


\section{Introduction}

Monte Carlo simulations of the track structure of ionising radiation in water medium are traditionally applied to estimate direct DNA damage (Francis et al., 2011; Friedland et al., 1998; Grosswendt, 2002; Nikjoo et al., 2006). Clustered damage occurring on short segments of the DNA (length of a few nanometres) is assumed to be the actuator for subsequent radiobiological effects, such as carcinogenesis or cell death (Goodhead, 2006; Khanna and Jackson, 2001). Track structure simulations are suitable for investigating clustered DNA damage, as they imitate the inherent stochastic nature of radiation transport by treating each single interaction of a particle and its secondaries in matter. In this way, the particle transport through the medium is simulated event-by-event, allowing a high spatial resolution in the order of the mean free path (nanometre scale). For this purpose, track structure simulations require input parameters in the form of cross sections for the interaction of charged particles with water molecules in the liquid phase. Water is commonly used to represent biological material, which is justified because water is the most abundant molecule present in biological cells. Investigations on the accuracy of using water to represent the DNA are currently being conducted at PTB (Bug et al., 2012). Due to the absence or unreliability of measured interaction cross sections in the condensed phase, track structure simulations generally utilise physical interaction cross sections for water that rely on measurements in water vapour, supplemented by extrapolations and theoretical models for condensed-phase effects (Dingfelder et al., 1998; Emfietzoglou and Nikjoo, 2005; Kutcher and Green, 1976; Rudd et al., 1992). The interaction between neighbouring molecules and the modification of the inter- and intra-molecular structure lead to significant changes of interaction cross sections, to energy shifts and a reduced amplitude of vibrational excitations as well as to additional collective modes of energy loss in the condensed phase compared to the gas phase (Michaud et al., 2003; Sanche et al., 1995). The existing cross section data for liquid water utilised in track structure simulations therefore contain an unknown bias.

Interaction cross sections of low-energy electrons (velocity is in the same order of magnitude as the velocity of bound electrons in water) have a potential additional unidentified bias due to higher order perturbation effects, which have to be approximated in theoretical calculations. The transport of low-energy secondary electrons is, however, most important as they are densely ionising (the mean distance between subsequent 
inelastic collisions is on the order of a few $\mathrm{nm}$ ) and therefore have a high probability to produce clustered DNA damage. Furthermore, a considerable number of electrons with a kinetic energy below $100 \mathrm{eV}$ is produced as secondary particles by any kind of ionising radiation.

A benchmarking test for track structure simulations of physical radiation interactions in water in the condensed phase was recently proposed by Toburen et al. (2010). They designed an experiment in which $6 \mathrm{MeV}$ protons were traversing a thin layer of amorphous solid water (ASW) and the energy spectra of secondary electrons with energies between about $1 \mathrm{eV}$ and $1 \mathrm{keV}$ were detected for different emission angles. In their measurements, the proton beam was pulsed (pulse duration $1 \mathrm{~ns}$, pulse rate $2 \mathrm{MHz}$ ) (Dingfelder et al., 2008). The emitted electrons were registered by a time-of-flight detector positioned at an adjustable angle with respect to the axis of the incident proton beam. Angles between $15^{\circ}$ and $60^{\circ}$ in the forward direction and between $115^{\circ}$ and $155^{\circ}$ in the backward direction were chosen. The $40 \mathrm{~nm}$ thick layer of ASW was frozen on a cryogenically cooled copper substrate of about $1 \mu \mathrm{m}$ thickness, where an amorphous structure was gained by the applied dosing method. The ASW density was estimated to be about $0.6 \mathrm{~g} / \mathrm{cm}^{3}$ and its potential surface roughness was assumed to have no significant effect on the secondary electron spectra. The absolute yield of secondary electrons was determined by normalising the integrated yield of the electron emission from a bare copper foil (integration with respect to emission angle and energy) to the published total yield of Koyama et al. (1981).

The stopping power as well as the secondary electron spectra of $6 \mathrm{MeV}$ protons in water can be realistically determined within the framework of the first Born approximation, as the proton velocity is sufficiently high. Moreover, the thickness of the copper foil and the ASW layer is small enough to negligibly change the stopping power and therefore the secondary electron spectra across the volume. The compliance of both criteria is essential to benchmark only the secondary electron transport in the simulations by the experimentally determined electron emission spectra.

The ASW layer was thick with respect to the range of the majority of emitted secondary electrons (the range of a $1 \mathrm{keV}$ electron is about $40 \mathrm{~nm}$ ) to eliminate any influence of the copper, ensuring that the measured emission spectra represent the secondary electron production and transport in water. The ASW layer was, 
however, thin enough to avoid a significant effect on the electron spectra by ASW charging (Dingfelder et al., 2008). As ASW in an insulator, this potential influence on the spectra would originate from the formation of a surface potential, affecting the subsequent emission of low-energy electrons by reducing their probability of emission and, presumably, their energy.

ASW is a suitable model for liquid water (which is used in track structure simulations) for two reasons: the similarity of the phonon bands and the structural similarity, which leads to the expectation that also ionisation and excitation cross sections are similar. A difference between the two states of water might, though, arise from thermal fluctuations, which are increased for the liquid state of water due to the higher temperature and the resulting higher amplitude of molecular vibrations (Michaud et al., 2003).

The measured angular secondary electron spectra have already been compared with those obtained by Monte Carlo simulations using PARTRAC where they showed good agreement for electron energies above about $100 \mathrm{eV}$, but below this energy the results from the simulation overestimated the electron yield up to a factor of 10 (Toburen et al., 2010).

This study aims to compare the results from Toburen et al. (2010) with the track structure codes PTra and Geant4-DNA, including also two different corrections for surface effects in both simulations.

\section{Methods}

\subsection{Interaction cross section data in PTra and Geant4-DNA}

In this work, PTra (version PTra-h2o-1103) and Geant4 (version 9.4, p. 02) were used. The PTB Track structure code PTra has been specifically developed for an application in nanodosimetry, where an accurate transport of low-energy secondary electrons on the nanometre scale is essential (Grosswendt et al. 2002). Geant4 is a general-purpose, open-source toolkit for the simulation of the passage of particles through matter 
that can, among other applications, also be used for track structure simulations by including the Geant4 Very Low Energy Extensions (Geant4-DNA) (Incerti et al., 2010).

Track structure simulations follow each primary particle and its secondaries event-by-event by random sampling of path length, type of interaction, energy loss, energy transferred to a secondary electron in case of an ionisation, as well as the scattering angle. The probability distributions used for random sampling are derived from the interaction cross sections of the medium with a specific type of charged particle. In the simulation, the particles are transported until their energy falls below a certain limit, generally given by the lower limit of the interaction cross section data, and are then assumed to deposit their remaining energy at the position of their last interaction. Detailed descriptions regarding the principle of track structure simulations can be found in Friedland et al. (1998), Grosswendt (2002), Zaider et al. (1994) as well as in Nikjoo et al. (2006), where also a comprehensive overview of existing codes is provided.

The interaction of $6 \mathrm{MeV}$ protons can be well described by scattering theories based on the first Born approximation. For such protons the target thickness is negligibly small compared to the range of the protons in water, so that they experience no significant energy loss and angular deflection. In addition, the cross section for charge transfer is negligible for protons of this energy. Ionisation interactions of those protons are treated in PTra by a semi-empirical model for the ionisation cross section developed by Rudd et al. (1992), which is based on experimental data of water vapour. Secondary electron spectra resulting from those ionisations and their angular distribution are determined by the Hansen-Kocbach-Stolterfoht (HKS) model, which uses the semi-classical approximation (Bernal and Liendo, 2006; Hansen and Kocbach, 1989). Geant4DNA treats ionisations by $6 \mathrm{MeV}$ protons within the framework of the first Born approximation (PWBA) and the dielectric formalism for liquid water (Incerti et al., 2010).

The intended benchmarking of secondary electron emission spectra relies on an accurate description of the production of secondary electrons by $6 \mathrm{MeV}$ protons in ASW, therefore figures 1 and 2 compare the energy spectra of those electrons as well as their distribution of scattering angles, obtained by PTra and Geant4DNA. The discrepancies in the energy spectra reflect the differences between the HKS model and the PWBA 
(ICRU, 1995) (figure 1). The yield of secondary electrons of energy above about $100 \mathrm{eV}$ is about a factor of 1.7 higher in PTra. The frequency of electrons between $9 \mathrm{eV}$ and $50 \mathrm{eV}$ is up to a factor of 1.25 higher when obtained by Geant4-DNA, continued by a decreasing distribution below $8 \mathrm{eV}$, whereas the frequency of secondary electrons continuously increases as their energy reduces in PTra.

The distribution of scattering angles obtained by both Monte Carlo codes exhibit scattering angles $\theta$ at or with a maximum at

$$
\cos \theta=\sqrt{E / E_{\max }}
$$

predicted by the binary encounter theory for a collision between a free charged proton of energy $T$ and a quasi-free target electron, producing a secondary electron with energy $E$, below the maximum energy in the free-electron limit

$$
E_{\text {max }}=4 \frac{m}{M} T
$$

where $m / M$ is the ratio of the electron to the proton mass. Due to a rather low momentum transfer to the target molecule in case of close collisions, the distribution for high-energy secondary electrons is strongly peaked, e.g. for $200 \mathrm{eV}$ secondary electrons the emission probability is highest at the predicted binary encounter peak of about $83^{\circ}$ (figure 2). In case of Geant4-DNA, the scattering angle is determined from the binary encounter theory and, in fact, always produces only a specific scattering angle for a specific electron energy. The HKS model used in PTra, on the other hand, produces a sharply peaked distribution of scattering angles for those electrons. For low-energy secondary electrons (below $100 \mathrm{eV}$ in Geant4-DNA), preferably produced in glancing collisions, the momentum transfer to the target becomes higher and the importance of the binding energy of the released electron increases. This results in an isotropic distribution of scattering angles in case of Geant4-DNA and a broader angular distribution according to the HKS model used in PTra with a maximum at the binary encounter peak.

Total electron ionisation cross sections in PTra are based on the energy loss functions derived by Dingfelder et al. (1998) and the semi-empirical model of Green and Sawada (1972) is used for a determination of the secondary electron energy after an ionisation as well as for excitation, as parameterised by Kutcher and Green (1976). Elastic scattering of electrons on water molecules is treated by the modified screened-Rutherford 
model for electrons of energy above $200 \mathrm{eV}$ (Grosswendt and Waibel, 1978) and the semi-empirical model of Brenner and Zaider (1983) below. A more detailed description of the interaction cross section data for water used in PTra is provided in Grosswendt et al. (2002) and Lazarakis et al. (2012).

As most electrons emitted from the ASW layer have energies below $10 \mathrm{eV}$ (Toburen et al., 2010; figure 10), the electron transport in PTra was extended from its previous limit of the ionisation threshold $10.79 \mathrm{eV}$ (Grosswendt, 2002) down to $1.7 \mathrm{eV}$. For this purpose, nine more phonon and vibrational excitation states and the formation of two transient anion states were considered in addition to each of the five ionisation and electronic excitation levels. The total cross sections for these additional physical processes were taken directly from Michaud et al. (2003). The energy loss for each level was determined by random sampling from a Lorentzian probability distribution function (PDF). The maximum of the PDF was at the mean excitation energy of each excitation state, provided by Michaud et al. (2003), and a full width half maximum as suggested by Dingfelder et al. (2008). Following the example of Dingfelder et al. (2008), the scattering angle after phonon and vibrational excitation were assumed to be the same as for elastic scattering, while dissociative attachment anion states were assumed to not lead to a change in direction. The angular deflection due to an electronic excitation was generally assumed to be negligible. The low-energy excitations were only applied in the range between $1.7 \mathrm{eV}$ and $100 \mathrm{eV}$, which is the energy range for which the experiments of Michaud et al. (2003) were conducted. The set of total interaction cross sections for water applied in PTra is shown in figure 3.

Detailed information on the interaction cross sections for electrons used in Geant4-DNA is provided in Incerti et al. (2010). Of the two choices provided for the treatment of elastic scattering, the 'G4DNAChampionElasticModel' was chosen as it also takes polarisation and exchange effects in the lowenergy domain into account. The lower energy limit of $4 \mathrm{eV}$ for elastic scattering was reduced to $2 \mathrm{eV}$, being aware that these cross sections would be extrapolated. Recently, the low-energy excitation cross sections from Michaud et al. (2003) were also included in the Geant4-DNA toolkit and were used for the simulation in this work (Francis et al., 2011). 


\subsection{Simulation setup}

It was necessary to perform two different kinds of simulations, the first one to investigate a potential impact of the copper foil on the secondary electron emission spectra and the second one to determine the angledependent emission spectra from the ASW.

In the first simulation, the impact of the copper foil on the proton energy and angular deflection as well as on the spectrum of secondary electrons, produced within the copper foil and reaching the adjacent ASW layer, was investigated by Monte Carlo simulation to confidently exclude any impact of the interactions occurring within the copper on the secondary electron spectrum emitted from the ASW. As neither PTra nor Geant4DNA possess a cross section data set that would enable track structure simulations in copper, the simulations were performed using the general Geant 4 toolkit with the 'Low Energy Electromagnetic Physics' (Chauvie et al., 2004), where the transport of protons and high energetic electrons (lower energy limit of $250 \mathrm{eV}$ ) is based on the stopping power and energy straggling as well as on multiple scattering theories for electron transport. The experimental setup, illustrated in figure 4, was represented in the Monte Carlo simulations by a water box of dimensions $1 \mu \mathrm{m} \times 1 \mu \mathrm{m} \times 40 \mathrm{~nm}$ and adjacent copper of dimensions $1 \mu \mathrm{m} \times 1 \mu \mathrm{m} \times 1 \mu \mathrm{m}$, both surrounded by vacuum. The density of the water was $0.6 \mathrm{~g} / \mathrm{cm}^{3}$, as given by Toburen et al. (2010). In the experiment, the cross sectional area of the target is very large compared to the range of primary and secondary particles scattered into an emission angle of $90^{\circ}$ to the primary beam. In the simulations, however, only the central part of the target with an arbitrary cross sectional area of $1 \mu \mathrm{m} \times 1 \mu \mathrm{m}$ was considered for both volumes to minimise computation time. This is compromised by a small probability for secondary electrons escaping the volume at angles around $90^{\circ}$ to the central beam axis. This probability is by at least a factor of 100 lower than for other emission angles and therefore negligible. As the surrounding volume was a vacuum, the protons were incident perpendicularly at the centre of the surface of the water box. At the back surface of the copper, the energy spectrum of protons and their spatial deviation with respect to the central axis of the beam was determined as well as the energy spectrum of secondary electrons, which would leave 
the copper in any direction. A total number of $10^{6}$ initial protons of $6 \mathrm{MeV}$ energy was used in the simulations.

In the second simulation, the secondary electron spectra emitted from the ASW were determined as a function of the emission angle for a $6 \mathrm{MeV}$ proton beam being incident normal at the centre of the surface of a water box of area $1 \mu \mathrm{m} \times 1 \mu \mathrm{m}$, thickness $40 \mathrm{~nm}$ and density $0.6 \mathrm{~g} / \mathrm{cm}^{3}$ by track structure simulations with PTra and Geant4-DNA, using $10^{6}$ protons for each simulation to achieve satisfactory statistics.

\subsection{Surface potential}

A surface potential was introduced in both PTra and Geant4-DNA simulations to account for charge accumulation effects of the ASW due to the ionisation of water molecules with a subsequent ejection of electrons. In this case, the escape probability for an electron depended on its energy and impact angle at the ASW/vacuum interface. As the exact mechanism of charge effects in the ASW was not clearly evident, two different simple cases were considered to investigate the influence of such a potential on the electron spectra. In the first case, it was assumed that the ionisations within the ASW lead to a positive potential barrier at the surface, through which low-energy electrons have a certain probability to tunnel (figure 5a). In the other case, a step-like surface potential was applied (figure 5b) to take into account that the ASW is an insulator, which will be charged due to the ionisations occurring within the target. An electron had to overcome a work function to be released into the vacuum, whereupon its energy was reduced accordingly. A similar assumption for the treatment of a surface barrier was made by Michaud et al. (2003), who assumed an in-bulk negative potential of the ASW relative to the vacuum and applied Snell's law, but did not consider an energy loss of the electron.

For both cases chosen to represent the surface potential, the probability of transmission was determined as a function of electron energy and impact angle on the surface. In the simulations, the decision whether an electron was transmitted or reflected at the surface was made by random sampling, using the transmission probability as the threshold criterion. In case of transmission, the diffraction angle was calculated by Snell's 
law; for reflection, the transport of the electron was continued within the ASW by using the reflection angle to determine the momentum direction for the subsequent part of the electron trajectory.

\subsubsection{Positive surface potential barrier}

The positive potential barrier was assumed to be located at the ASW/vacuum interface with arbitrary height $V_{0}$ and width $a$. To solve the time-independent Schrödinger equation, a plane-wave approach was used, distinguishing between three regions: region 1 in the ASW, where the wave function is given by the superposition of incoming and reflected waves, region 2 within the potential barrier, where the wave function describes the superposition of the waves transmitted into the potential barrier and the waves reflected within the potential barrier and region 3 in the vacuum where the wave function describes the transmitted wave.

Due to the requirement of conservation of the total energy and the parallel component of the wave vectors with respect to the surface $\left(k_{1 p}=k_{2 p}=k_{3 p}\right)$, the wave vectors normal to the surface were determined by

$$
\begin{aligned}
& k_{1 n}=\sqrt{\left|\vec{k}_{1}\right|^{2}-k_{3 p}^{2}}=\hbar^{-1} \sqrt{2 m_{e f f} E-2 m E \sin ^{2} \beta} \\
& k_{2 n}=\sqrt{\left|\vec{k}_{2}\right|^{2}-k_{3 p}^{2}}=\hbar^{-1} \sqrt{2 m_{e f f}\left(E-V_{0}\right)-2 m E \sin ^{2} \beta}
\end{aligned}
$$

where $k_{2 n}$ is real for $E \geq V_{0}$ and imaginary for $E<V_{0}$, and

$$
k_{3 n}=\sqrt{\left|\vec{k}_{3}\right|^{2}-k_{3 p}^{2}}=\hbar^{-1} \sqrt{2 m E} \cos \beta .
$$

$E$ is the energy of the electron incident on the surface and $V_{0}$ is the height of the potential barrier. By increasing the electron mass $m$ by a factor $X_{m}>1.0$, the resulting effective mass $m_{\mathrm{eff}}=m X_{\mathrm{m}}$ accounts for a polarisation of the molecules of the medium, which arises from the electron travelling through the ASW. In the simulation, this factor allows for diffraction effects at the boundary between the ASW and the vacuum and is required to achieve a realistic angular distribution after the diffraction. The emission angle $\beta$ was determined by Snell's law, which leads to

$$
\cos ^{2} \beta=1-X_{m} \sin ^{2} \alpha
$$


where $\alpha$ is the incidence angle on the surface. Retaining continuity of the wave function and its derivatives at each boundary, the probability for transmission of an electron of energy $E$ and incidence angle $\alpha$ on the potential barrier is

$$
T=1-R
$$

where the probability for reflection $R$ is given by

$$
R=\frac{\left(k_{1 n}-k_{3 n}\right)^{2}+\left(k_{2 n}^{2}-k_{1 n}^{2}-k_{3 n}^{2}+\left(k_{1 n} k_{3 n} / k_{2 n}\right)^{2}\right) \sin ^{2}\left(k_{2 n} a\right)}{\left(k_{1 n}+k_{3 n}\right)^{2}+\left(k_{2 n}^{2}-k_{1 n}^{2}-k_{3 n}^{2}+\left(k_{1 n} k_{3 n} / k_{2 n}\right)^{2}\right) \sin ^{2}\left(k_{2 n} a\right)}
$$

Examples of the transmission probability as a function of $E$ are shown in figure 6 . The resonances originate from the interference of multiple reflections of the wave function of the propagating electron within the barrier. The transmission maxima correspond to the situation when the width of the barrier accommodates an integer multiple of half the De-Broglie wavelength $\lambda$ of the electron, such that for normal incidence $=n \lambda / 2$. In this case, the sine-term in eq. (8) becomes zero as $k_{2 n} a=n \pi$ and the transmission probability reaches unity (for $X_{m}=1$ ). The energies of the transmission maxima for the different resonance modes $n$ of the barrier are therefore depending on the height and width of the barrier as well as on the impact angle. For increasing impact angle, the transmission probability decreases under the influence of the factor $X_{\mathrm{m}}$ for the effective electron mass, even for electrons of energies much higher than the height of the potential barrier. With increasing height $V_{0}$, the first maximum of the transmission probability is shifted to higher energies; with increasing barrier width $a$, the frequency of the resonance maxima increases.

\subsubsection{Potential step at the ASW surface}

The step function was located at the ASW/vacuum interface, corresponding to the work function in solid state physics, which describes the minimum energy needed to remove an electron from the Fermi level. The timeindependent Schrödinger equation was solved by applying a plane-wave approach, i.e. a superposition of the incoming and reflected waves in the ASW region and the transmitted wave in the vacuum. The application of the conservation laws of total energy and of parallel momentum to the surface and the requirement of continuity of the wave functions and its derivatives at the boundary lead to solutions for the ratios of the 
amplitudes of the reflected or transmitted wave to the incoming wave. The probabilities for reflection $R$ and transmission $T$ are then

$$
R=\left(\frac{k_{1 n}-k_{2 n}}{k_{1 n}+k_{2 n}}\right)^{2}, T=1-R=\frac{4 k_{1 n} k_{2 n}}{\left(k_{1 n}+k_{2 n}\right)^{2}}
$$

where the wave vectors for the region in the ASW $k_{1 n}$ and of the waves transmitted to the vacuum $k_{2 n}$ represent the component of the wave vectors normal to the surface

$$
k_{1 n}=\hbar^{-1} \sqrt{2 m E} \cos \alpha, k_{2 n}=\hbar^{-1} \sqrt{2 m(E-\phi)} \cos \beta
$$

where $m$ is the electron mass, $E$ the energy of the electron when incident on the surface, $\phi$ the work function, $\alpha$ the incidence angle on the surface and $\beta$ the emission angle, determined from Snell's law by

$$
\cos ^{2} \beta=1-\frac{E}{E-\phi} \sin ^{2} \alpha
$$

After emission, the energy of the electron was reduced by the work function $\phi$.

The probabilities of transmission and reflection are shown in figure 7 exemplarily for a work function of $\phi=$ $3 \mathrm{eV}$, for the impact angle $\alpha$ averaged over all emission directions, as well as for $\alpha=0^{\circ}$ and $45^{\circ}$. Electrons of energy below $3 \mathrm{eV}$ are not able to leave the ASW. Below an energy of about $10 \mathrm{eV}$ there is a significant probability (>3\%) for reflection at the surface, which depends strongly on the impact angle. After experiencing a reflection, the transport of the electron is continued in the ASW. For the average impact angle, the transmission probability is higher than the reflection probability for an electron of energy above about 4.2 $\mathrm{eV}$. Similarly, in case of an impact angle of $0^{\circ}$, the transmission probability is above 0.5 for electrons with energy above about $3.1 \mathrm{eV}$. For larger impact angles, however, the transmission probability will be zero up to a threshold energy $E_{t h}=\phi /\left(1-\sin ^{2} \alpha\right)$ (as can be seen from eq. 11) with a subsequent steep rise.

\section{Results and discussion}

\subsection{Impact of the copper foil}


As expected from the stopping power according to the Bethe-Born theory and the thickness of the target, the energy of the incident $6 \mathrm{MeV}$ protons changed negligibly after their passage through the water and copper targets; the mean energy of the transmitted protons was $5.964 \mathrm{MeV}$ (figure 8a). This energy corresponds well to the energy loss calculated from the stopping power of $6 \mathrm{MeV}$ protons in copper and liquid water available in the PSTAR data base (PSTAR, 2011). Less than $0.1 \%$ of the protons leave the copper layer with an energy below $5.930 \mathrm{MeV}$ or above $5.981 \mathrm{MeV}$. The spatial deviation of the protons at the back surface of the copper was within $100 \mathrm{~nm}$, with the probability being less than $0.1 \%$ for a deviation exceeding $15 \mathrm{~nm}$ (figure $8 \mathrm{~b}$ ). which is negligible when compared to the $\mathrm{Cu}$ foil thickness of approximately $1 \mu \mathrm{m}$ (Toburen et al. 2010).

Figure 9 shows the yield of secondary electrons produced by a traversing proton that would enter the adjacent ASW layer after leaving the copper foil. According to equation (2), the binary encounter theory predicts a maximum secondary electron energy of $12 \mathrm{keV}$, which is clearly visible by the truncation of the spectrum at this energy. The decreasing emission spectrum below $1 \mathrm{keV}$ might be an artefact from the multiple-scattering theories applied in this simulation; it would be expected from scattering theory and also due to the transport and subsequent energy loss of higher energy electrons that the number of secondary electrons per energy interval of energy below $1 \mathrm{keV}$ is higher than above. Nonetheless, taking into account that the thickness of the ASW of $40 \mathrm{~nm}$ is approximately equal to the range of $1 \mathrm{keV}$ electrons, those results provide evidence that the yield of electrons with an energy high enough to cross the ASW layer or to produce further secondary electrons, which could leave the ASW, is negligible as is therefore their contribution to the electron emission spectra from the ASW. This means that the setup of the second simulation, using only the water layer to determine the electron emission spectra, is sufficient for a comparison to the measured data.

\subsection{Secondary electron spectra}

Figure 10 shows the energy spectra obtained from the experiment of Toburen et al. (2010) and simulations by means of Geant4-DNA (G4) and PTra with different low-energy limits applied. The experimental data for the single differential yield of emitted secondary electrons were obtained from the measured angle-resolved 
energy spectra by numerical integration, assuming that the yields at $0^{\circ}$ and $180^{\circ}$ equal those at $15^{\circ}$ and $155^{\circ}$ and that the yield at $90^{\circ}$ is zero. The uncertainty of the measured electron yields is lower than $30 \%$ for electron energies between $3 \mathrm{eV}$ and $100 \mathrm{eV}$ and a factor 2 below $3 \mathrm{eV}$ (Toburen et al. 2010).

The extension of the energy limit in PTra from the ionisation threshold of $10.79 \mathrm{eV}$ down to $1.7 \mathrm{eV}$ by including low-energy excitations and dissiociative attachment anion states (and still accounting for electronic excitations and elastic scattering below the ionisation threshold), leads to a smooth continuation of the electron emission spectra to lower energies. The spectra obtained via Geant4-DNA without applying the lowenergy excitation and electron attachment cross sections exhibit a kink at about $9 \mathrm{eV}$, which is the lower energy limit of the electronic excitation cross sections. In this case, electrons of energy below $9 \mathrm{eV}$ undergo only elastic scattering in the simulations. This is, of course, physically inaccurate and usually the lower energy limit of the simulation would be set at this energy when using older Geant4-DNA versions, where this was the only available interaction cross section set below $9 \mathrm{eV}$. By including all available interaction cross sections, the qualitative energy spectra are similar to those obtained by PTra but show a kink at about $11 \mathrm{eV}$ which could be due to the lower threshold of the ionisation process.

Above about $50 \mathrm{eV}$, the measured energy spectra are in good agreement with those of PTra and Geant4DNA, with the exception that above $100 \mathrm{eV}$ the energy spectra obtained by Geant4-DNA are about $60 \%$ lower. This could be due to the sudden decrease in the spectrum at about $100 \mathrm{eV}$, the origin of which still has to be investigated as it seems not to be of physical origin. It might result from the abrupt limit of the lowenergy excitation cross sections at this energy, which was, however, also present in the low-energy excitation cross sections applied in PTra. An Auger peak is visible in the spectra from PTra at about $500 \mathrm{eV}$, resulting from the ionisation of the oxygen K-shell (Kaplan et al., 1990). Auger electron emission was not yet included in this version of Geant4-DNA, but is not essential for this work. Below $50 \mathrm{eV}$, the measured and simulated energy spectra deviate by up to a factor of 4 (PTra) or 7 (Geant4).

The generally larger slope of the energy spectra obtained by means of Geant4-DNA compared to those of PTra is likely due to the differences in electron production by the protons (see figure 1). 
In figure 11, the influence of a surface barrier on the emission energy spectra determined by the simulations is exemplarily shown for two configurations of each barrier model. Including a potential step of height $\phi$ in both simulations, PTra and Geant4-DNA, improves the agreement with the experimental data. Compared to the spectra obtained without the potential step, a shift to lower energies according to $\phi$ by $2.5 \mathrm{eV}$ or $3 \mathrm{eV}$ is observed. Furthermore, a relatively lower yield of low-energy electrons is obtained, as the probability of reflection at the surface increases with decreasing electron energy. For PTra, the agreement for a potential step of $2.5 \mathrm{eV}$ or $3 \mathrm{eV}$ is better, leading to deviations of the simulated and measured data much lower than the experimental uncertainties, except in the region between about $6 \mathrm{eV}$ to $8 \mathrm{eV}$ where the simulated data are up to $45 \%$ higher. Using Geant4-DNA, a potential step of $3.5 \mathrm{eV}$ yields a better agreement, but still with differences up to a factor of 3 compared to the measured yield in the region between $5 \mathrm{eV}$ and $15 \mathrm{eV}$.

Using a positive potential barrier instead of the work function does not seem to be appropriate, as, with reasonable parameters for the height $V_{0}$ and width $a$ of the barrier, only the yield of electrons of very low energy (about $4 \mathrm{eV}$ for $V_{0}=3 \mathrm{eV}$ and about $6 \mathrm{eV}$ for $V_{0}=4 \mathrm{eV}$ ) was found to be reduced. The width of the barrier has a greater influence than the height, leading to a strong reduction of particles below the energies stated above. The choice of the parameter $X_{\mathrm{m}}$, accounting for the increase of the projectile's mass due to polarisation of the surrounding ASW molecules, has a minimal influence on the spectra if chosen between 1.0 and 1.5 as is shown in figure $11 \mathrm{a}$.

Even when a potential step was applied in the simulations, the energy spectra obtained in different emission angles are qualitatively similar and do not reproduce the shift of the maximum yield of secondary electrons to lower energies, as the emission angle approaches the forward or backward direction (figure 12). This effect was interpreted by Toburen et al. (2010) to originate from a surface potential where the transmission probability depends on the incidence angle normal to the surface.

A direct comparison of the double differential yield of secondary electrons obtained by simulations with the measured data of Toburen et al. (2010) is shown in figure 13, exemplarily for emission angles of $15^{\circ}$ in the forward direction and $135^{\circ}$ in the backward direction. To improve the readability, the graphs obtained from 
the simulations were re-binned to smoothen the curve and an uncertainty was estimated from the statistical fluctuations. Without considering a surface potential, the deviation of the calculations by means of PTra from the measured data is up to a factor of 4 for energies below about $10 \mathrm{eV}$, but significant improvement can be obtained by including a potential step of $2.5 \mathrm{eV}$. In this case, the simulated data are in good agreement with the measured data within the uncertainties of the experiment (as stated above), again, except in the region between $6 \mathrm{eV}$ and $8 \mathrm{eV}$, where the simulated spectra are about a factor of 1.5 higher than the experimental spectra. This might be due to an overestimation of the number of secondary electrons produced after an ionisation or to an underestimation of the excitation cross section (where the energy would be reduced with regard to the excitation level) relative to the elastic scattering cross section (where the electron is assumed to be scattered without energy loss). A similar effect can be observed in the energy spectra obtained by Geant4DNA, which differ from the measured data up to a factor of 7 when no surface potential is used in the simulations, and agree generally well in case of a $3.5 \mathrm{eV}$ potential step, except in the region between 5 and 10 $\mathrm{eV}$, where the simulated data are still up to a factor 3 higher than the measured energy spectra.

\section{Conclusion}

Angle-dependent secondary electron emission spectra induced by $6 \mathrm{MeV}$ protons in amorphous solid water were measured by Toburen et al. (2010) and used in this work to benchmark the secondary electron transport in the track structure Monte Carlo codes PTra and Geant4-DNA. Without the application of a surface potential in the simulations, the double differential electron yield obtained by means of Geant4-DNA is similar to the results obtained by PARTRAC (Toburen et al. 2010), while the energy spectra from PTra show a slightly better agreement with the measured data. Good agreement of double and single differential secondary electron yields between measured and simulated data was reached within the experimental uncertainties by including a potential step at the ASW surface into the track structure simulations.

Even though the agreement of the simulation results with the measured spectra is generally good in the case investigated in this work, the unknown bias of the interaction cross section data, potentially affecting results of track structure simulations, should be kept in mind. 


\section{Acknowledgement}

The authors thank Larry Toburen for providing the measured data and details on the measurements.

\section{References}

Bernal, M. A., Liendo, J. A., 2006. The HKS model for electron production in liquid water by light ions. Nuc. Instrum. Meth. B 251, 171-176.

Brenner, D. J. , Zaider, M., 1983. A computationally convenient parameterisation of experimental angular distributions of low energy electrons elastically scattered off water vapour. Phys. Med. Biol. 29, 443-447.

Bug, M. U., Baek, W. Y., Rabus, H., 2012. Simulation of ionisation clusters formed in nanometric volumes of the deoxyribose-substitute tetrahydrofuran. Int. J. Radiat. Biol. 88, 137-142.

Dingfelder, M., Hantke, D., Inokuti, M., Paretzke, H. G., 1998. Electron inelastic-scattering cross sections in liquid water. Radiat. Phys. Chem. 53, 1-18.

Dingfelder, M., Travia, A., McLawhorn, R. A., Shinpaugh, J. L., Toburen, L., 2008. Electron emission from foils and biological materials after proton impact. Rad. Phys. Chem. 77, 1213-1217.

Francis, Z., Incerti, S., Capra, R., Mascialino, B., Montarou, G., Stepan, V., Villagrasa. C., 2011. Molecular scale track structure simulations in liquid water using the Geant4-DNA Monte-Carlo processes. Appl. Radiat. Isot. 69, 220-226.

Friedland, W., Jacob, P., Paretzke, H. G. and Stork, T., 1998. Monte Carlo simulation of the production of short DNA fragments by low-linear energy transfer radiation using higher-order DNA models. Radiat. Res. $150,170-182$.

Geant4, 2011. http://geant4.web.cern.ch/geant4/.

Goodhead D T., 2006. Energy deposition stochastics and track structure: what about the target? Radiat. Prot. Dosim. 122, 3-15.

Green, A. E. S., Sawada. T., 1972. Ionisation cross sections and secondary electron distributions. J. Atm. Terr. Phys. 34, 1719-1728.

Grosswendt, B., 2002. Formation of ionisation clusters in nanometric structures of propane-based tissueequivalent gas or liquid water by electrons and $\alpha$-particles. Radiation and Environmental Biophysics 41, 103112.

Grosswendt, B., Waibel, E., 1978. Transport of low energy electrons in nitrogen and air. Nuc. Instrum. Meth. $155,145-156$.

Hansen, J. P., Kocbach, L., 1989. Ejection angles of fast delta electrons from K-shell ionisation induced by energetic ions. J. Phys. B 22, L71-L77.

Incerti, S., Ivanchenko, A., Karamitros, M., Mantero, A., Moretto, P., Tran, H. N., Mascialino, B., Champion, C., Ivanchenko, V. N., Bernal, M. A., Francis, Z., Villagrasa, C., Baldacchino, G., Gueye, P., Capra, R., Nieminen, P., Zacharatou, C., 2010 Comparison of GEANT4 very low energy cross section models with experimental data in water. Med. Phys. 37, 4692-4708. 
International Commission on Radiation Units and Measurements. 1995. ICRU Report 55 Secondary electron spectra from charged particle interactions.

Kaplan, I. G., Miterev, A. M., Sukhonosov, V. Y., 1990. Simulation of the primary stage of liquid water radiolysis. Radiat. Phys. Chem. 36, 493-498.

Khanna, K. K., Jackson, S. P., 2001. DNA double-strand breaks: signaling, repair and the cancer connection. Nature Genetics 27, 247-254.

Koyama, A., Shikata, T., Sakairi, H., 1981. Secondary electron emission from Al, Cu, Ag, and Au, by proton impact. Jpn. J. Appl. Phys. 20, 65-70.

Kutcher, G. J., Green, A. E. S., 1976. A model for energy deposition in liquid water. Radiat. Res. 67, 408425.

Lazarakis, P., Bug, M. U., Gargioni, E., Guatelli, S., Rabus, H., Rosenfeld A. B., 2012. Comparison of nanodosimetric parameters of track structure calculated by the Monte Carlo codes Geant4-DNA and PTra. Phys. Med. Biol. 57, 1231-1250.

Michaud, M., Wen, A., Sanche, L., 2003. Cross sections for low-energy (1-100 eV) electron elastic and inelastic scattering in amorphous ice. Radiat. Res. 159, 3-22.

Nikjoo H., Uehara, S., Emfietzoglou, D., Cucinotta, F. A., 2006. Track-structure codes in radiation research. Radiat. Meas. 41, 1052-1074.

PSTAR. 2011. Stopping power and range tables for protons. NIST, http://physics.nist.gov/PhysRefData/Star/Text/PSTAR.html

Rudd, M. E., Kim, Y.-K., Madison, D. H., Gay, T. J., 1992. Electron production in proton collisions with atoms and molecules: energy distributions. Rev. Mod. Phys. 64, 441-490.

Sanche, L., Märk, T. D., Hatano, Y., 1995. Low energy electron interaction with condensed matter. In: International Atomic Energy Agency IAEA. Atomic and molecular data for radiotherapy and radiation research. ISSN 1011-4289.

Toburen, L. H., McLawhorn, S. L., McLawhorn, R. A., Carnes, K. D., Dingfelder, M., Shinpaugh, J. L., 2010. Electron emission from amorphous solid water induced by passage of energetic protons and fluorine ions. Radiat. Res. 174, 107-118.

Zaider, M., Vracko, M. G., Fung, A. Y. C., Fry, J. L., 1994. Electron transport in condensed water. Radiat. Prot. Dosim. 52, 139-146. 


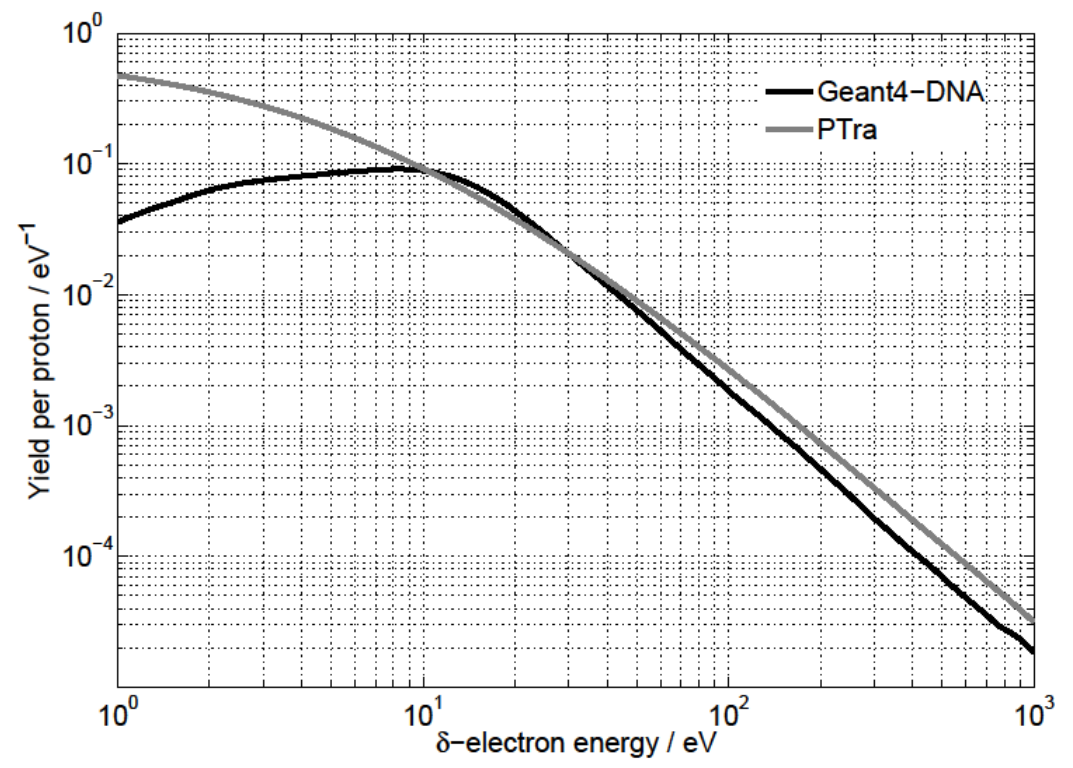

Figure 1. Energy spectra of $\delta$-electrons produced directly by a $6 \mathrm{MeV}$ proton passing through a $40 \mathrm{~nm}$ thick water layer. The spectra were calculated by PTra and Geant4-DNA track structure simulations and show the energy spectra integrated over the scattering angles.

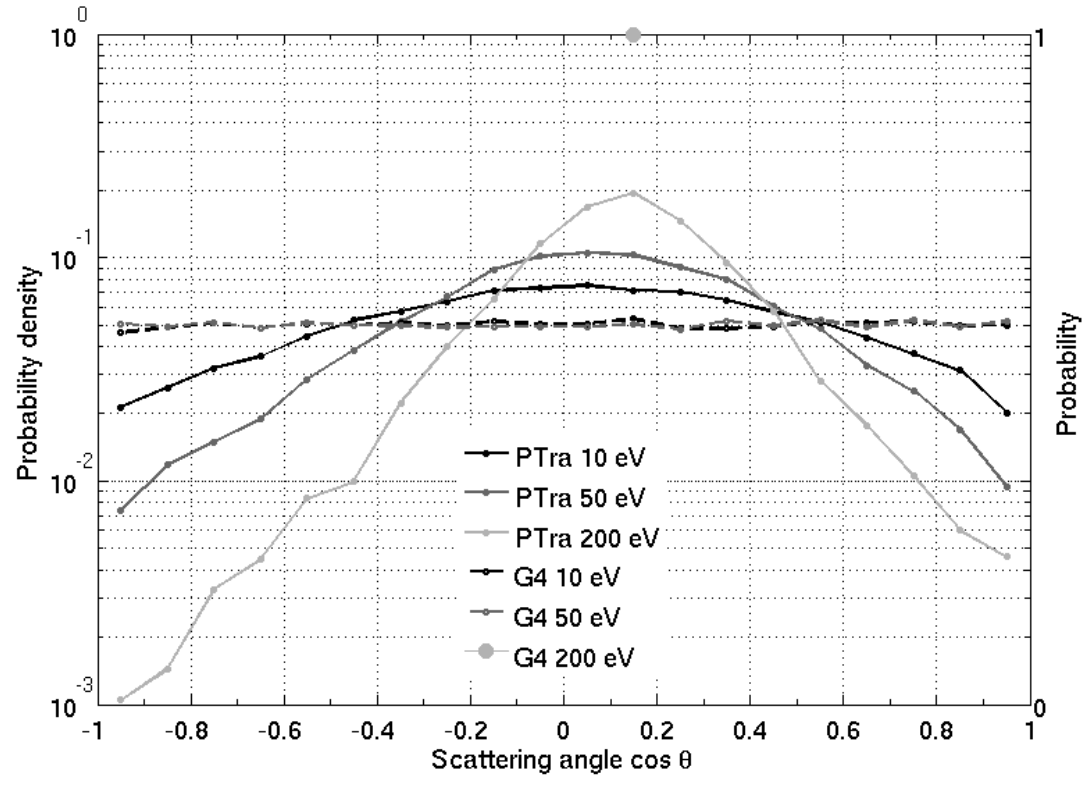

Figure 2. Probability density distribution of the cosine of the scattering angle $\theta$ of secondary electrons with energies $10 \mathrm{eV}, 50 \mathrm{eV}$ and $200 \mathrm{eV}$ exemplarily calculated by PTra and Geant4-DNA Monte Carlo simulations. For $200 \mathrm{eV}$ secondary electrons in Geant4-DNA, a discrete scattering angle is calculated from the binary encounter theory (right hand y-axis, symbol: circle). 


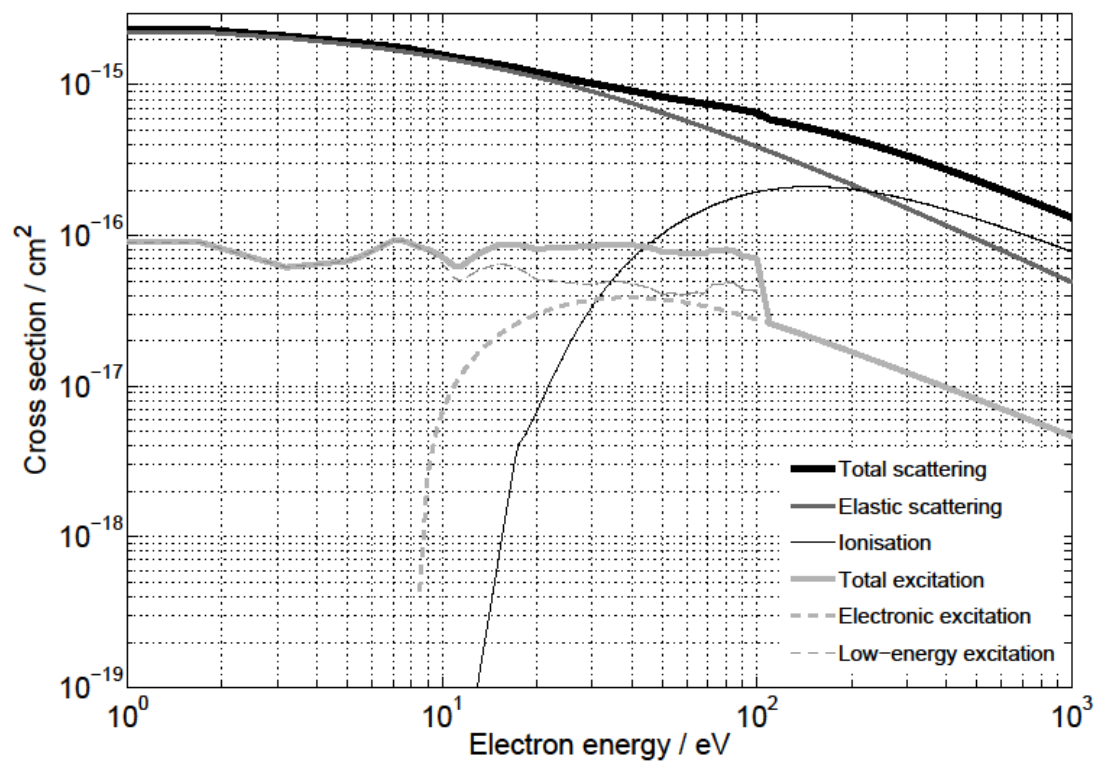

Figure 3. Total cross sections of water for electrons used in PTra for total scattering, elastic scattering, ionisation and excitation including the cross sections for low-energy excitation of Michaud et al. (2003).

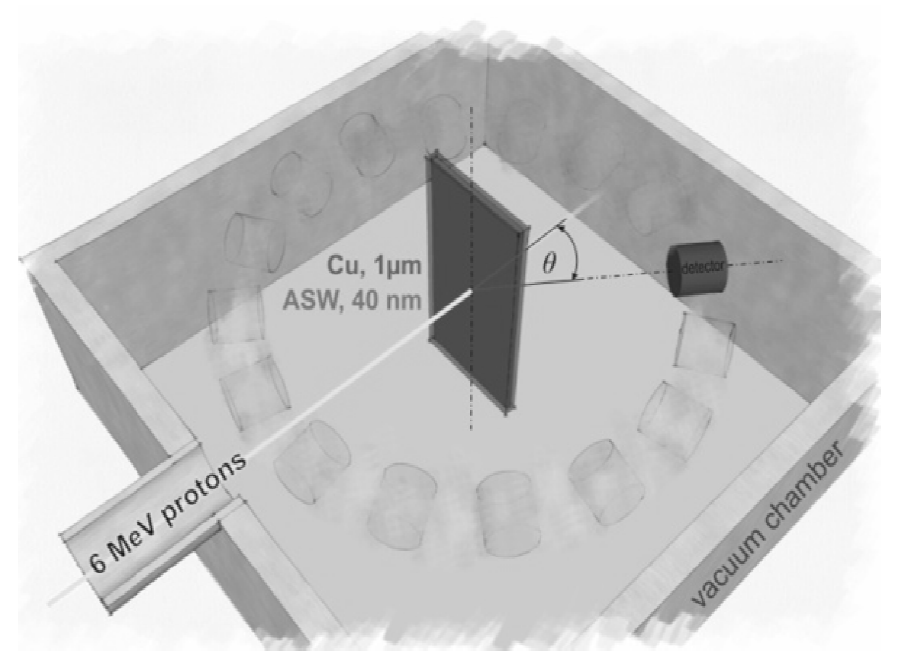

Figure 4. Geometrical setup for experiment and simulations: $6 \mathrm{MeV}$ protons were incident on a $40 \mathrm{~nm}$ thick slab of ASW deposited on both sides of a copper foil. Spectra of secondary electrons leaving the ASW into the surrounding vacuum were determined as a function of emission angle. 

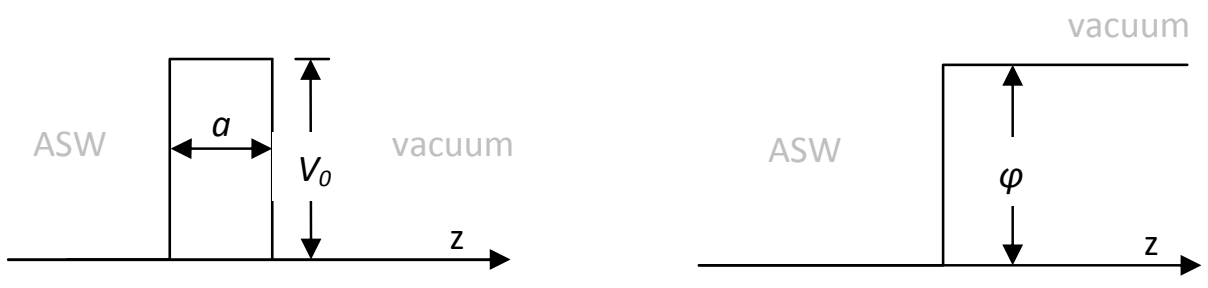

Figure 5. Sketch of the surface potentials assumed in this work. (a) shows the positive potential barrier of height $V_{0}$ and width $a,(b)$ the step-like potential including a work function of height $\phi$.

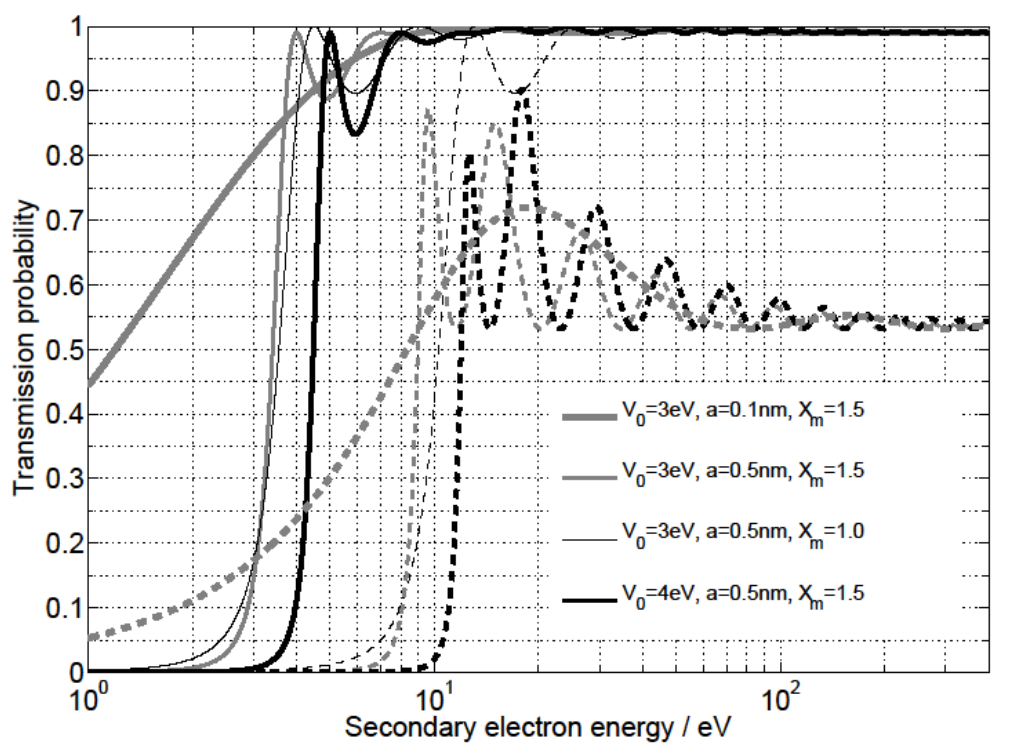

Figure 6. Transmission probability as a function of electron energy for different angles of incidence $\alpha=0^{\circ}$ (solid lines) and $\alpha=54^{\circ}$ (dashed lines), illustrated for three different strengths of the potential. 


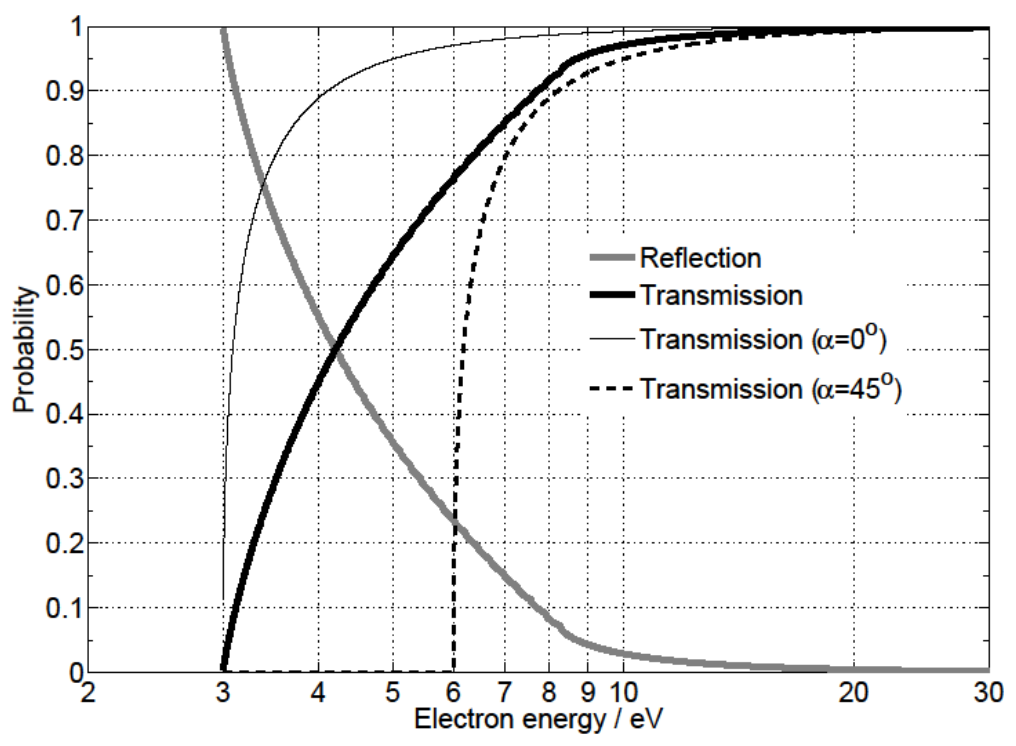

Figure 7. Probability for transmission and reflection as a function of electron energy for a work function of 3 eV shown exemplarily for the impact angle $\alpha$ averaged over all emission directions, as well as for $\alpha=0^{\circ}$ and $45^{\circ}$.
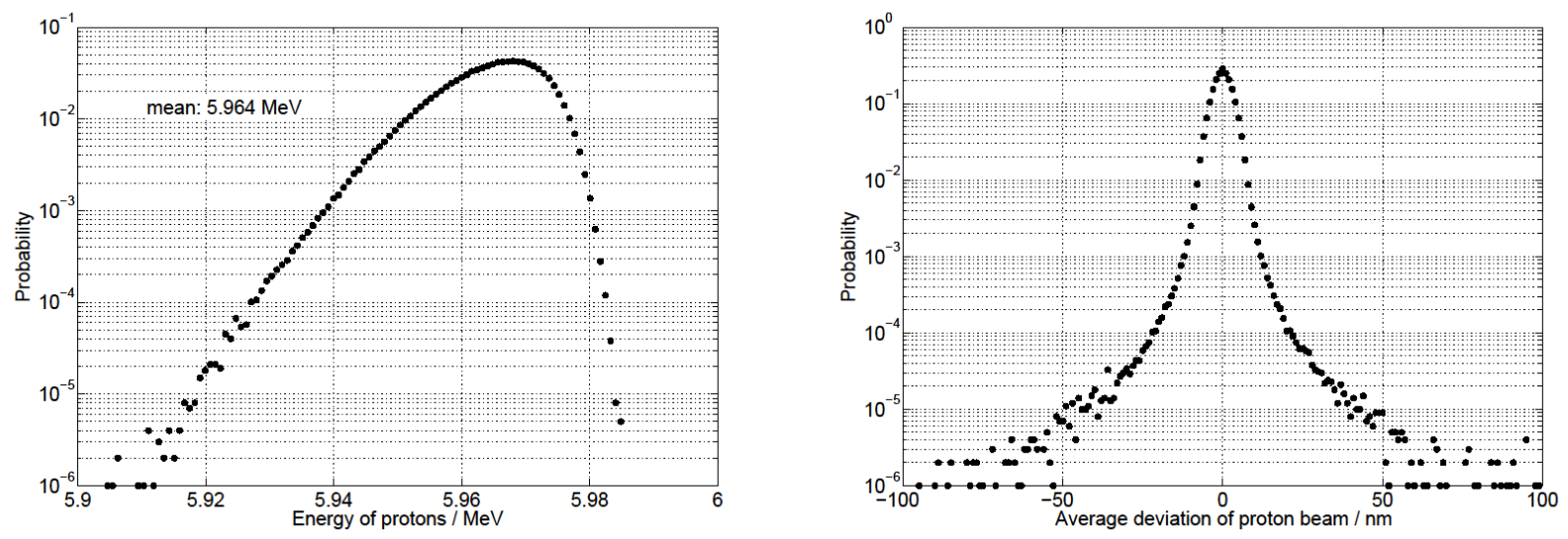

Figure 8. Probability distribution of energy (a) and spatial deviation from the beam's central axis (b) of an incident $6 \mathrm{MeV}$ proton beam after passing through a $40 \mathrm{~nm}$ thick layer of water and 1 rm of copper. 


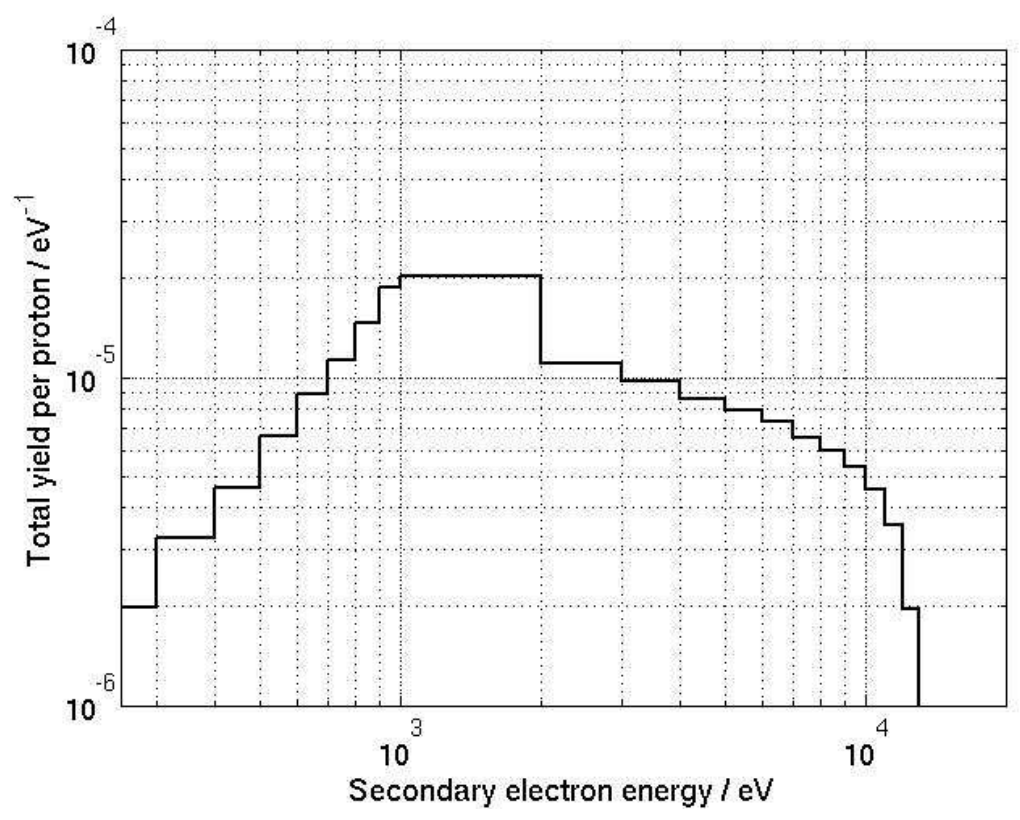

Figure 9. Energy spectrum of secondary electrons produced by a $6 \mathrm{MeV}$ proton beam and leaving the back surface of the copper layer.

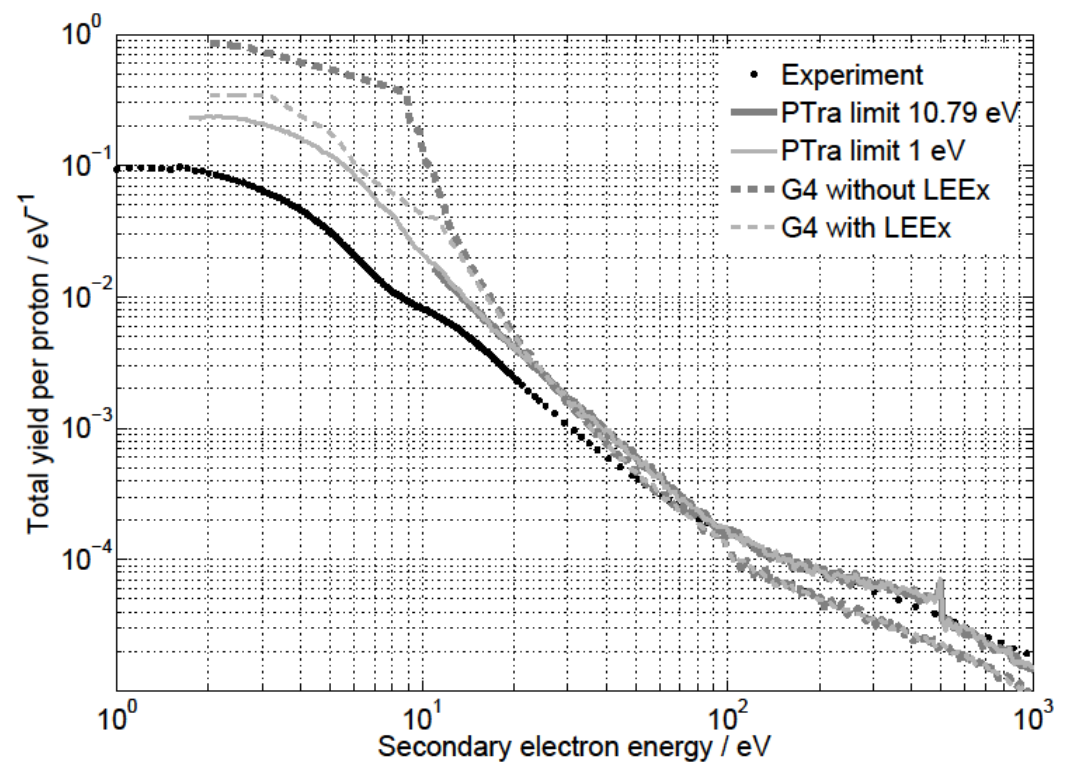

Figure 10. Secondary electron energy spectra obtained from experiment and Monte Carlo simulations using Geant4-DNA and PTra with and without low-energy excitation cross sections (LEEx). 

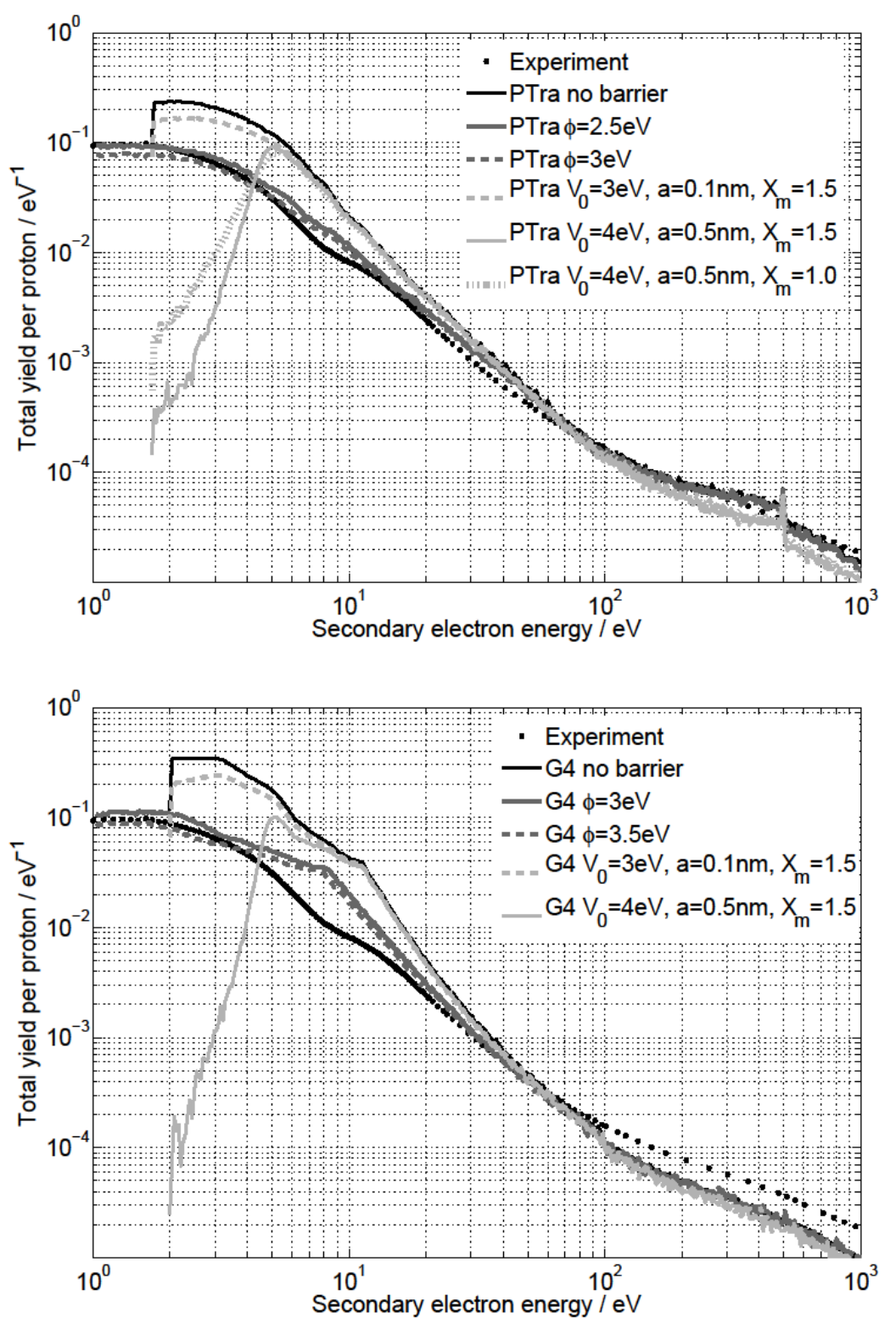

Figure 11. Single differential yield of secondary electrons as a function of their energy obtained by integrating measured and simulated angle-resolved spectra over the emission angle. The simulations were performed using PTra (a) and Geant4-DNA (b). 

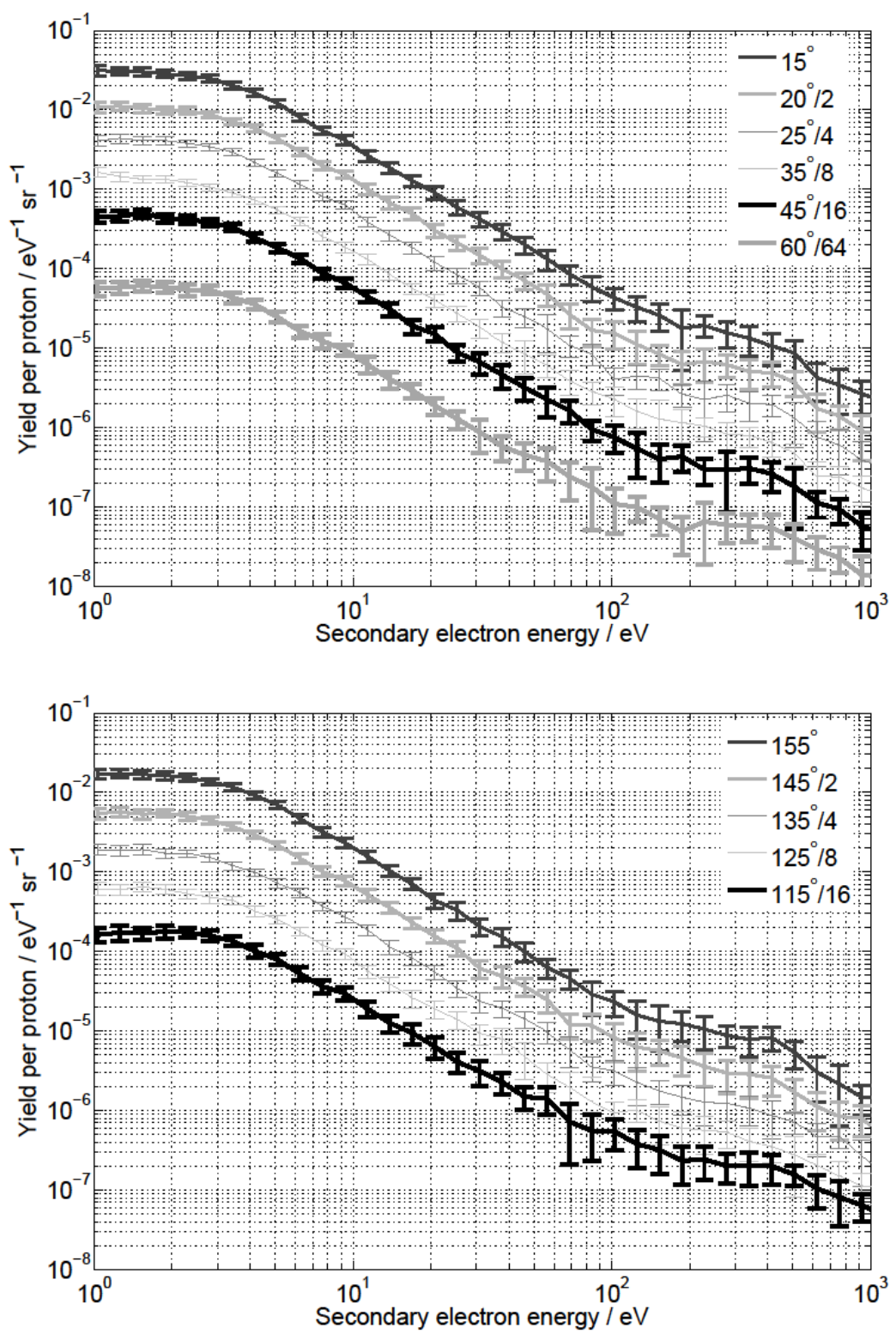

Figure 12. Double differential yield of secondary electrons with respect to the solid angle of emission and energy obtained by means of PTra, assuming a potential step at the ASW surface of $2.5 \mathrm{eV}$ for forward (a) and backward (b) emission angles. 

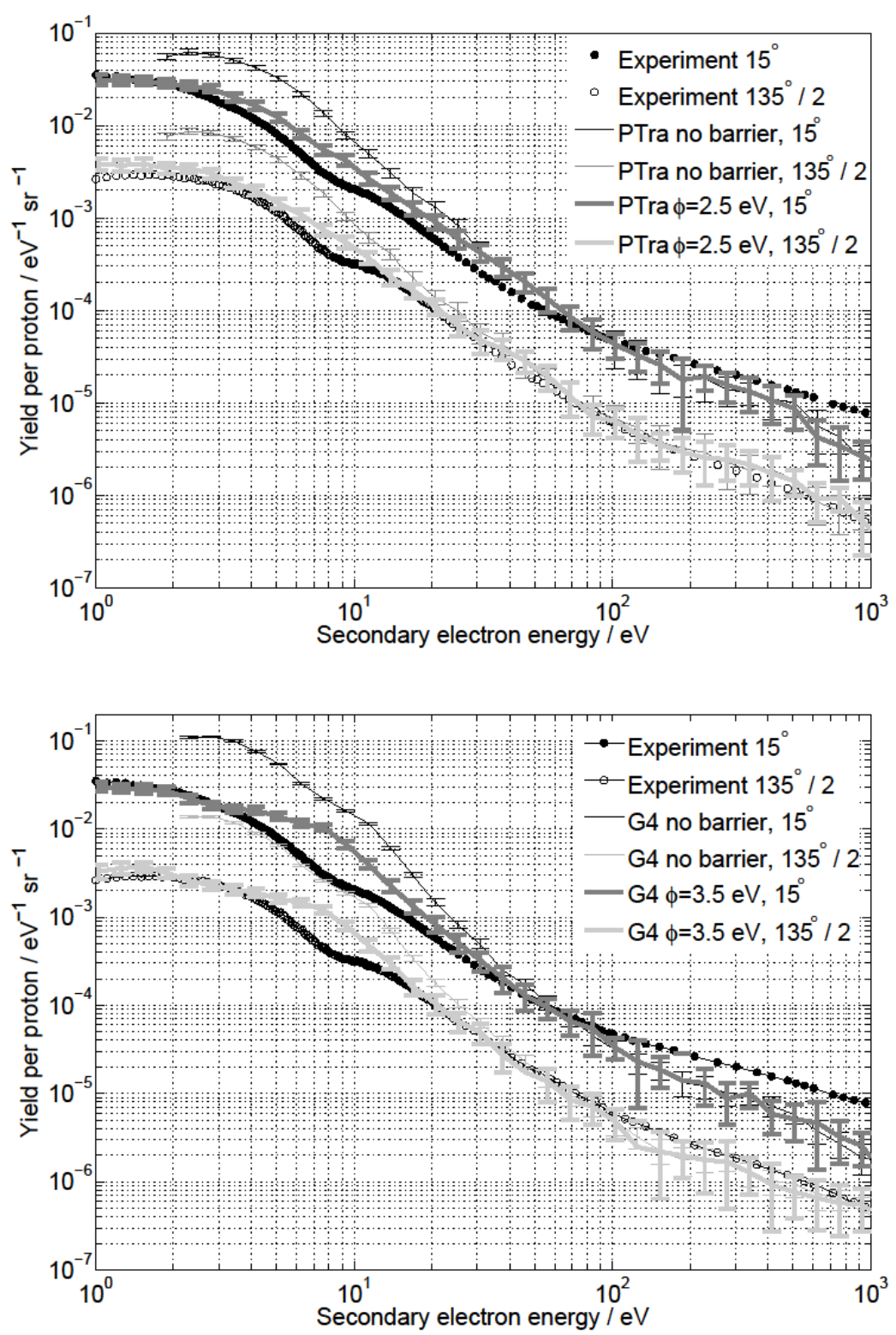

Figure 13. Secondary electron spectra obtained from the experiment of Toburen et al. (2010) and simulations using PTra (a) and Geant4-DNA (b) for selected forward and backward emission angles. 